\section{Formaldehyde toxicology - occupational versus environmental HAP?}

As a ubiquitous air pollutant in both indoor and outdoor air the potential adverse health effects from formaldehyde exposure have been subject to numerous critical public health reviews by regulatory agencies. Public health advisories have, however, been based primarily on the linear extrapolation of dose-response data from occupational studies where repeated elevated levels of exposure have led to both respiratory irritation and sensitisation as well as probable increased incidences of nasopharyngeal cancers.

In this paper the inadequacies in the interpretation of occupationally derived dose-response relationships to derive safe levels for public exposure to a key HAP in the absence of a detailed understanding of dose-response mechanisms are explored. Issues around the 2004 IARC reclassification of formaldehyde as Class 1 'carcinogenic to humans' are discussed and the technical background as to why public cancer incidence rates from formaldehyde exposure have historically been overestimated by a number of international regulatory agencies. The latest knowledge of formaldehyde toxicology and carcinogenesis is discussed to illustrate the limitations in simple linear extrapolation of occupational data to derive cancer risk estimates and safe exposure limits for assessment of ambient air quality monitoring data and public health risk assessment. Occupational and ambient limits for formaldehyde in South Africa are tabled for review and debate.

Keywords: HAPs, formaldehyde toxicology, dose-response models, occupational versus environmental health risk assessment.

\section{Introduction}

Formaldehyde is a ubiquitous air pollutant that has been the focus of significant scientific inquiry and regulatory debate over the last decade. Inconclusive or conflicting epidemiological studies coupled with incorrect risk estimates from doseresponse models have led to significant confusion and misrepresentation of the risk profile of formaldehyde both in terms of occupational and public health management.

In this paper the key issues and current state of knowledge of formaldehyde toxicology are discussed within the context of assessing health risks from both occupational and environmental exposures. The limitations of applying statistically based dose-response extrapolations to toxico- logical data sets to development of health risk estimates and safe exposure levels in the absence of adequate understanding of biological mechanisms of toxicity and dose-response behaviour are illustrated and appropriate limits for occupational and ambient air quality in South Africa are proposed based on latest scientific knowledge.

\section{Formaldehyde as an air pollutant}

Formaldehyde is the most common naturally occurring aldehyde in the environment. It is an essential metabolic intermediate in all cells, both human and animal, and is produced endogenously. Formaldehyde is, however, also generated by a range of anthropogenic sources including automotive exhaust, cigarette smoke and various products containing formaldehyde-based resins and glues used in the manufacture of particle board and plywood.

Occupational exposures occur in a wide variety of settings and industries which can be summarised into three main circumstances, namely (i) production of aqueous formaldehyde (formalin) and its use in chemical industry (eg resins, preservative etc.), (ii) in relation to its release from formaldehyde-based resins in which it is present as a residual and/or through the hydrolysis and decomposition by heat namely during manufacture of wood products, textiles, synthetic vitreous insulation and plastics, and (iii) in relation to the pyrolysis or combustion of organic matter, namely engine exhaust gases or during fire fighting.

Environmental, or non-occupational, exposures to airborne formaldehyde, range from around $0.5 \mathrm{ug} / \mathrm{m3}$, which represents the mean natural background concentration (WHO, 2001) to up to $100 \mathrm{ug} / \mathrm{m} 3$ as short term peak levels recorded in heavy traffic or during severe inversions in urban environments. The levels of formaldehyde in indoor air are often higher than those in outdoor air due to a variety of anthropogenic sources, notably offgassing of urea-formaldehyde foam insulation, particle board and formaldehydebased resins. Mean levels in homes with no urea-formaldehyde foam insulation range from 25 to $60 \mathrm{ug} / \mathrm{m} 3$ whereas levels in mobile homes have been recorded over $100 \mathrm{ug} / \mathrm{m} 3$ (IARC, 1995).

Formaldehyde is formed in the troposphere by photochemical oxidation of many types of organic compounds from both natural and anthropogenic sources. Given the diversity and abundance of formaldehyde precursors in urban air, secondary atmospheric formation frequently exceeds direct emissions from combustion sources, especially during photochemical air pollution episodes.

Formaldehyde is not a persistent air pollutant being highly reactive with photochemicaly generated hydroxyl radicals, undergoing direct photolysis, as well as rapid hydrolysis on contact with water vapour. Atmospheric half-life is strongly dependent on intensity of sunlight, temperature and humidity/moisture content but is no longer than a few hours to days in most climatic conditions. 


\section{Scientific paper}

Table 1 - Summary of levels at which adverse health effects are observed (after WHO, 2001)

\section{Health effects from formaldehyde exposure}

Most formaldehyde exposures occur by inhalation or by skin/eye contact. Due to its rapid solubility in water, exposure to formaldehyde vapours produces local irritation in mucous membranes including eyes, nose, throat and upper respiratory tract.

Low-dose acute exposures can result in headache, rhinitis, and dyspnea whilst higher doses may cause severe mucous membrane irritation, burning and lacrimation, and lower respiratory effects such as bronchitis, pulmonary oedema or pneumonia. Dermal irritation and burns can occur, with potential for contact dermatitis in sensitized individuals. The exact mechanism of action of toxicity is not clear, but it is known that it can interact with molecules on cell membranes and in body tissues and fluids and disrupt cellular functions (WHO, 2001). The levels at which adverse/irritant effects are observed are summarised in Table 1.

In terms of chronic exposures the major concerns associated with repeated formaldehyde exposure are sensitization and cancer. In sensitised individuals, formaldehyde can cause asthma and contact dermatitis.

There is sufficient evidence from experimental animals and in vitro test systems to demonstrate carcinogenic and genetoxic effects from formaldehyde (Inchem, 2002). Development of nasal carcinomas in rats exposed to formaldehyde vapours has been demonstrated. DNA protein cross-links, chromosomal aberrations (CA), sister chromatid exchange (SCE) and gene mutation have all been demonstrated in humans cells in vitro. The frequencies of CA, SCE and micronuclei

in peripheral blood lymphocytes have been demonstrated to have been affected in studies among workers exposed to formaldehyde (Shaham, 2007). The available evidence clearly demonstrates that there exists the potential for formaldehyde to be a possible human carcinogen. However, epidemiological evidence for a causal association between formaldehyde exposure and upper respiratory tract cancers in humans is limited or conflicting and the matter remains at this time the subject of significant scientific and regulatory policy debate as discussed below.

\section{Hazard Classification and Carcinogenicity}

In terms of managing the risks associated with hazardous chemicals, hazard classification, which serves to define whether under any defined circumstances, a substance could result in a one or more adverse health effects, such as for example cancer, is based primarily on a weight-ofevidence approach.

In June 2004 the International Agency for Research on

\begin{tabular}{|l|l|l|}
\hline $\begin{array}{l}\text { Concentration } \\
\left(\mathbf{m g} / \mathbf{m}^{3}\right)\end{array}$ & Exposure Time & $\begin{array}{l}\text { Health effects in } \\
\text { general population }\end{array}$ \\
\hline 0.18 & Repeat exposure & $\begin{array}{l}\text { Odour deflection } \\
\text { threshold }\left(50^{\text {th }}\right. \\
\text { percentble) }\end{array}$ \\
\hline 0.1 to 3.1 & Single or repeated & $\begin{array}{l}\text { Throat and nose } \\
\text { irritation threshold }\end{array}$ \\
\hline $0.6-1.2$ & Single or repeated & Eye irritation threshold \\
\hline $0.5-2$ & $3-5$ hours & $\begin{array}{l}\text { Decreased nasal } \\
\text { mucous flow }\end{array}$ \\
\hline $5-6.2$ & 30 minutes & $\begin{array}{l}\text { Tolerable for 30 } \\
\text { minutes with } \\
\text { lacrymation }\end{array}$ \\
\hline $12-25$ & 1 hour & Strong lacrymation \\
\hline $37-50$ & - & $\begin{array}{l}\text { Pulmonary oedema, } \\
\text { pneumonia, danger } \\
\text { to life }\end{array}$ \\
\hline
\end{tabular}

Cancer (IARC) changed its hazard classification for formaldehyde from a "probable" to "known" human carcinogen.

At the time of the IARC change in classification, most other regulatory bodies and agencies such as the EU, USEPA, NIOSH, and ACGIH still regarded formaldehyde as a probable or possible human carcinogen based on sufficient data in animals but limited or conflicting evidence in humans. The IARC reclassification rather than prompting a shift to more stringent classification by other regulatory bodies, initiated more intense review and evaluation of existing cohort data sets. This re-analysis of the data has led to questions in terms of appropriate hazard classification for formaldehyde as well as a re-interpretation of the dose-response relationship and risk estimates as detailed later in this paper.

The IARC re-classification was based on the decision that a statistically significant excess of deaths from NPC in workers exposed to formaldehyde had been demonstrated in comparison to the US national population, with statistically significant exposure-response relationships for peak and cumulative exposures (IARC 2006).

The IARC decision has, however, been challenged (Marsh et el 2005, Marsh et al 2007, Bosetti et al 2007) as it was deemed to have been based primarily on the results reported by Hauptmann et al $(2003 ; 2004)$ from an updated 1994 follow-up of the National Cancer Institute's (NCl) cohort mortality study of workers exposed to formaldehyde (Blair et al., 1986; Blair et al., 1990).

The 2003 Hauptmann et al report, which focused on lymphohematopoietic malignancies, included an indication of a causal association between formaldehyde exposure and mortality from leukaemia, particularly myeloid leukaemia. The association was immediately questioned on grounds of biological implausibility and the methods applied to the exposure assessment and statistical analysis (Casanova et al 2004). The 2004 Hauptmann et al report, which focused on solid tumors suggested a possible causal association between formaldehyde exposure and cancer of the nasopharynx (NPC). As with leukaemia, the $\mathrm{NCl}$ exposure-response findings for formaldehyde and NPC were based exclusively on internal mortality rate comparisons and statistically significant exposure-response relationships were observed for only two of four formaldehyde exposure metrics considered, namely peak and cumulative formaldehyde exposure. More importantly, the majority of the NPCs examined in the $\mathrm{NCl}$ cohort were observed in one plant.

The above nothwithstanding, the IARC judgement on Hauptmann et al (2004) was that (i) the estimated doseresponse relationships were quite certain, (ii) that it is improbable that that all of the positive findings for NPC 
As with leukaemia, the NCl exposure-response findings for formaldehyde and NPC were based exclusively on internal mortality rate comparisons and statistically significant exposureresponse relationships were observed for only two of four formaldehyde exposure metrics considered, namely peak and cumulative formaldehyde exposure. More importantly, the majority of the NPCs examined in the $\mathrm{NCl}$ cohort were observed in one plant.

reported in epidemiological studies could be explained by bias or unrecognised confounding effects, and (iii) that the results could be generalised to other settings with formaldehyde exposure. Hence the IARC decision to reclassification formaldehyde.

However, subsequent reanalysis of the $\mathrm{NCl}$ cohort data by Marsh \& Youk (2005), indicated that the data does not support the causal association between formaldehyde exposure and NPC as suggested by Hauptmann et al. (2004). More specifically, sensitivity analysis demonstrated considerable uncertainty in the exposure-response relationships, and that the NPC mortality excesses observed in the one plant reported in the $\mathrm{NCl}$ data were not associated with formaldehyde but rather may reflect the influence of nonoccupational risk factors.

In a comprehensive review of cancer in industry workers and professionals exposed to formaldehyde as detailed in cohort studies up to 2006, Bosetti et al (2007), reported no appreciable excess risk for oral and pharyngeal, sinonasal or lung cancers. Bosetti et al (2007) acknowledged the non-significantly increased relative risk for nasopharyngeal cancer among industry workers attributable to a cluster of deaths in a single plant as detailed in the $\mathrm{NCl}$ cohort data set but concluded insufficient evidence to support a casual association between formaldehyde exposure and NPC.

On the basis of the re-evaluation of available cohort data, Marsh et al (2007) have suggested that the IARC decision to classify formaldehyde as Group 1 (known human carcinogen) should be reconsidered. No formal response has been issued by the IARC at this time.

\section{Dose-Reponse Relationships}

The US EPA Integrated Risk Information System (IRIS) lists an inhalation unit risk for formaldehyde of $1.3 \times 10-5$ per $\mathrm{ug} / \mathrm{m} 3$. This unit risk estimation results in a predicted increased risk of developing cancer of 1 in 10,000 from inhalation exposure to formaldehyde at levels of approximately $0.008 \mathrm{mg} / \mathrm{m3}$. Comparison of this level against the levels reported earlier in this paper for urban outdoor air and workplace and home indoor air concentrations indicates that in terms of this risk factor, cancer incidences from formaldehyde in the general population would be predicted to be greater than 1 in 10,000 to 1 in 1,000. Cancer incidences for formaldehyde exposure from mobile home indoor air would be predicted to be greater than 1 in 100. These incidences are not supported by epidemiological data for NPC which is rare in most populations world wide with incidences per 100,000 of between 2 in USA white males aged 60 to 65 and 50 in Hong Kong chinese males of similar age group (Adami, 2007).

The US EPA inhalation risk was based on a simple linear extrapolation of a dose-response curve defined from a study by Kerns et al (1983) on rats and, despite the clear lack of congruence between risk model predictions and epidemiological data, a revision has not been formally issued since its release in 1987.

In 1991 the US EPA reevaluated its risk assessment for formaldehyde and calculated two revised risk estimates based on a linear risk estimation procedure but incorporating information on delivered dose. These estimates were between 6 and 50 times lower than the original 1987 estimates but were never finalised by the US EPA.

The significant over estimations in formaldehyde cancer risks for formaldehyde exposure by the US EPA stems from use of inappropriate risk assessment methods and dose-response projections. Dose-response relationships for carcinogenic compounds have traditionally been treated as non-threshold responses with extrapolations being made from relatively high dose-response data available from occupational type settings to very low level exposures applicable to public health settings. A detailed study of formaldehyde toxicological, mechanistic and dosimetric data was undertaken by the Chemical Industry Institute of Technology (CIIT) that evaluated dose-response relationships and risk estimation models for formaldehyde based on over 20 years of research data (CIIT, 1999; Conolly, 2004).

The CIIT analysis replaced conservative assumptions based on linear extrapolations and a simple non-threshold model for carcinogenic compounds with a biologically-based approach that recognises that for chemicals such as formaldehyde, which humans are exposed to low levels on a daily basis as part of normal cellular metabolism and that forms a normal component of metabolism with multiple pathways existing for its conversion, "standard" risk-assessment methods result in incorrect projections of the dose-response curve. According to Conolly (2004) the fundamental shift in approach relates to recognising the presence of a cytotoxicity threshold for formaldehyde in the range 0.6 to $1 \mathrm{ppm}$ that represents a de minimis (negligible risk of 10-6 or less) in terms of cancer risk associated with inhalation exposures.

In terms of dose-response relationships, the evidence of a cytoxicity threshold means that statistically based linear extrapolations of the doseresponse data to low exposure levels are invalid, and non-threshold approaches to formaldehyde risk modelling are not supported by available scientific evidence. The available information demonstrates that formaldehyde exposure only poses a carcinogenic hazard under conditions that both induce toxicity and cause sustained regenerative proliferation.

The CIIT model has been subjected to peer review by both the US EPA and Health Canada, with both regulatory agencies agreeing that the model provided a significant improvement over default approaches adopted in previous assessments.

Whilst the US EPA has not withdrawn their original risk estimate from the IRIS database, a notice has been issued with a revised estimate for inhalation unit risk of $5.5 \mathrm{x}$ 
The significant over estimations in formaldehyde cancer risks for formaldehyde exposure by the US EPA stems from use of inappropriate risk assessment methods and dose-response projections. Dose-response relationships for carcinogenic compounds have traditionally been treated as non-threshold responses with extrapolations being made from relatively high dose-response data available from occupational type settings to very low level exposures applicable to public health settings.

10-9 per $\mathrm{ug} / \mathrm{m} 3$. This revised risk estimate is orders of magnitude below the original estimate of 1987, and reflects the limitations of linear dose-response extrapolations made in the absence of supporting test data and inadequate knowledge of mechanistic toxicity behind the dose-response relationship. As of 2008 the US EPA is currently preparing an updated toxicological review and IRIS summary for formaldehyde that is due to be issued before the end of the year.

\section{Implications for occupational and environmental risk management}

From a public perspective, the current regulatory guidance on assessment and management of health risks associated with formaldehyde inhalation exposures must appear confusing and contradictory. The reclassification of formaldehyde by IARC to Group 1 in 2004 suggests that more stringent levels of control and management of formaldehyde as a hazardous substance are imperative and need to be implemented. None of the other key regulatory agencies have however reclassified the hazard status of formaldehyde pending further clarity from the scientific community in respect of epidemiological data.

In contrast to the IARC reclassification, the reanalysis of dose-response relationships and biologically-based approach to risk estimations, indicates that the risks from exposure to formaldehyde is substantially lower than previously estimated, with the risks to the general public from average environmental exposure levels are negligible. Hazard classification and unit risk estimations represent two different components of risk assessment and management and despite first appearances, the current position is not contradictory

From an occupational health risk management perspective, the recognition of a cytotoxic threshold for carcinogenic response represents an important point of departure and de minimis level for regulation of worker exposures. Internationally, there remains a wide range in occupational limits set from $0.02 \mathrm{mg} / \mathrm{m3}$ (NIOSH) to 2.5 $\mathrm{mg} / \mathrm{m} 3$ (UK) with the trend being to reduce 8 hour TWA's to below the lower limit cytotoxic threshold value of 0.7 $\mathrm{mg} / \mathrm{m3}$.

The South African OHS Act TWA-OEL is currently the same as the UK at $2.5 \mathrm{mg} / \mathrm{m} 3$ and also requires revision in line with current scientific knowledge. The EU Scientific Committee on Occupational Exposure Limits (SCOEL) has proposed an 8 hour TWA of $0.25 \mathrm{mg} / \mathrm{m} 3$, which is an order of magnitude lower and is under consideration for EU member states that have limits above this level. A similar proposal for revision to the UK limits is being considered based on the SCOEL recommendations.

In terms of setting ambient air quality limits, the $\mathrm{WHO}$ (2001) has recommended an air quality guideline of 0.1 $\mathrm{mg} / \mathrm{m} 3$ as a 30 minute average for protection of general population. This is well below the lower limit for cytotoxic damage and potential carcinogenic risk but takes into account the need for protection against sensory irritant effects for which a NAOEL of $0.5 \mathrm{mg} / \mathrm{m} 3$ has been reported (Triebig et al, 2007).

On the basis of current scientific knowledge of formaldehyde toxicity and dose-response relationships, it is proposed that an appropriate South Africa limit for occupational exposures would be $0.7 \mathrm{mg} / \mathrm{m} 3$

(8-hr TWA), with recognition that the international trend is to reduce limits to $0.3 \mathrm{ppm}(0.37 \mathrm{mg} / \mathrm{m} 3)$, and that the guideline for ambient air quality should be set at a level of $0.1 \mathrm{mg} / \mathrm{m} 3$.

\section{References}

Adami HO. 2007. 'The enigmatic epidemiology of NPC'. In Proceedings of the Formaldehyde Int. Science Conference, Sept 2007, Barcelona.

Blair A, Stewart PA, O'Berg M, et al. 1996. 'Mortality among industrial workers exposed to formaldehyde.', J. National Cancer Inst. 76:195- 215.

Blair A, Saracci R, Stewart PA, et al. 1990. 'Epidemiological evidence on the relationship between formaldehyde exposure and cancer'. Scandinavian J. Work Environment and Health. 16:381-393.

Bosetti C, McLaughlin JK, Tarone RE, Pira E, La Vecchia C. 2007. Formaldehyde and cancer risk: a quantitative review of cohort studies through 2006. Annals of Oncology online publication Sept. 25.

Casanova M, Cole P, Collins JJ, Conolly R, Delzell E, Heck Hd HA, Leonard R, Lewis R, Marsh $\mathrm{GM}$, Sorsahan T. 2004. Comments on, 'Mortality from lymphohematopoietic malignancies among workers in formaldehyde industries'. J. National Cancer Inst. 16:96(12), 966-967. CIIT, 1999. 'Formaldehyde: Hazard characterization and dose-response assessment for carcinogenicity by the route of inhalation'. Chemical Industry Institute of Technology. Conolly RB, Kimbell JS, Janszen D, Schlosser PM, Kalisak D, Preston J, Miller FJ. 2004. 'Human respiratory tract cancer risks of inhaled formaldehyde: dose-response predictions derived from biologically-motivated computational modelling of a combined rodent and human dataset'. Toxicol. Sci. 82(1):279-296

Hauptmann M, Lubin JH, Stewart PA, Hayes RB, Blair A. Blair A. 2003. 'Mortality from lymphophematopoietic malignancies among workers employed in formaldehyde industries'. J. National Cancer Inst. 95:1615-1623.

Hauptmann M, Lubin JH, Stewart PA, Hayes RB, Blair A. Blair A. 2004. 'Mortality from solid cancers among workers in formaldehyde industries. American J.Epidemiology. 159:11171130.

IARC Monograph. 1995. 'Formaldehyde - In: Wood Dust and Formaldehyde'. Int. Agency for Research on Cancer. Vol 62.

IARC Monograph. 2006. 'Formaldehyde'. Int. Agency for Research on Cancer. Vol 88 Kerns WD, Pavkov KL, Donfrio DJ, Gralla EF, Swenberg JA. 1983. 'Carcinogenicity of formaldehyde in rates and mice after long-term inhalation exposure'. Cancer Res. 43:43824392.

Marsh GM, Youk AO, Morfield P. 2007. 'Misspecified and non-robuts mortality risk models for nasopharyngeal cancer in the National Cancer Institute Formaldehyde Worker Cohort Study'. Regulatory Toxicology and Pharmacology 47:59- 67.

Marsh GM, Youk AO. 2007. 'Re-evaluation of mortality risk from nasopharyngeal cancer in the formaldeyhyde cohort study of the National Cancer Institute'. Regulatory Toxicology and Pharmacology, 42:275-283, 2005

Shaham J. 2007. 'DPC formation and genetotoxicity in humans occupationally exposed to formaldehyde'. In Proceedings of the Formaldehyde Int. Science Conference, Sept 2007, Barcelona.

Triebig G, Bruckner Th, Lang I. 2007. 'Snesory irritation of formaldehyde in humans at occupational exposure limit value'. In Proceedings of the Formaldehyde Int. Science Conference,Sept 2007, Barcelona.

WHO Air Quality Guidelines 2nd Ed. 2001. 'Chapter 5.8 - Formaldehyde'. World Health Organisation, Denmark.

WHO, 2002. Concise International Chemical Assessment Document (CICAD). World Health Organisation, Geneva. Vol 40. 